\title{
Das Wirken des Leiters des sächsischen Ministeriums für Volksbildung Arthur Göpfert 1935 bis 1945
}

\author{
von \\ KONSTANTIN HERMANN
}

\begin{abstract}
„Statisten in Uniform“ nannte Joachim Lilla sein biografisches Nachschlagewerk über die Reichstagsabgeordneten der nationalsozialistischen Zeit. ${ }^{1}$ Diesen ,Statisten“ als politische Staffage, als willige Ausführende von Ordern der Reichs- und Landesregierungen beziehungsweise Gauleitungen, hat sich die Forschung in den vergangenen Jahren intensiver gewidmet. Im Fokus standen dabei vor allem die Durchsetzung und Etablierung der NS-Herrschaft in den Ländern und in der Wehrmacht, die Beteiligten am Holocaust, aber vor allem die regionalen und lokalen Ausprägungen des, braunen Totalitarismus' durch Nationalsozialisten der ,zweiten Reihe‘. Von umfangreichen Kompendien dazu, wie sie zum Beispiel Baden-Württemberg mit dem Band „Die Führer der Provinz" besitzt, ${ }^{2}$ ist die landesgeschichtliche Forschung in Sachsen jedoch noch entfernt. Perspektivisch wird hier die biografische Datenbank des Instituts für Sächsische Geschichte und Volkskunde Abhilfe schaffen können, die „Sächsische Biografie“. 3 Doch die kritische Beurteilung von regionalen Protagonisten der NS-Bewegung, die zunächst die biografische Erforschung voraussetzt, hat auch in Sachsen deutlich zugenommen. Diese Personen im ,zweiten Glied', sowohl in der Gauleitung, in der Landesregierung oder in kommunaler Verantwortung, werden zunehmend auch als Akteure, nicht mehr nur als Ausführende höherer Befehle in der Forschung wahrgenommen.

Mustergültige landesgeschichtliche Studien gibt es in zunehmender Zahl aus den vergangenen Jahren. ${ }^{4} 2011$ legte Mike Schmeitzner eine Monografie über Mutschmann vor, die aus vorher nicht genutzten Quellen aus sowjetischen Archiven schöpft. Das Buch konnte nach nur kurzer Zeit bereits in der zweiten Auflage veröffentlicht werden. ${ }^{5}$ Gemeinsam mit Gerhard Naser und Christine Pieper gab Schmeitzner im Januar 2012 den Band „Braune Karrieren“ heraus, der erstmals die Biografien aller sächsischen Minister in der NS-Zeit und weiterer Protagonisten der sächsischen NSDAP beinhaltet. ${ }^{6}$ Weitere biografische Projekte zu Nationalsozialisten, ,Mitläu-
\end{abstract}

1 Jonchim Lilla, Statisten in Uniform. Die Mitglieder des Reichstags 1933-1945. Ein biographisches Handbuch, Düsseldorf 2004.

2 Michael Kissener/Johchim Scholtyseck, Die Führer der Provinz. NS-Biographien aus Baden und Württemberg, Konstanz 1999.

3 Vgl. Sächsische Biografie, hrsg. vom Institut für Sächsische Geschichte und Volkskunde, www.isgv.de/saebi.

4 Mike Schmeitzner/Andreas Wagner (Hg.), Von Macht und Ohnmacht. Sächsische Ministerpräsidenten im Zeitalter der Extreme 1919-1952, Beucha 2006.

5 Mike Schmeitzner, Der Fall Mutschmann. Sachsens Gauleiter vor Stalins Tribunal, Beucha 2011.

6 Christine Pieper/Mike Schmeitzner/Gerhard Naser (Hg.), Braune Karrieren. Dresdner Täter und Akteure im Nationalsozialismus, Dresden 2012. 
fern' und Opfern befinden sich in Vorbereitung. Gerhard Lindemann und der Verfasser des vorliegenden Beitrags werden eine Aufsatzsammlung mit ausgewählten Biografien von Pfarrern der evangelisch-lutherischen Landeskirche Sachsens herausgeben, die alle kirchenpolitischen Richtungen und sächsischen Regionen berücksichtigen wird.

Der Hauptgrund für die frühere Zurückhaltung der Forschung dürfte in der in Diktaturen typischen beschnittenen Entscheidungskompetenz und den zu geringen Gestaltungsmöglichkeiten der zweiten Führungsebene liegen, die anscheinend nur wenige Spielräume bietet. Dies gilt umso mehr für das nationalsozialistische Regime mit seinem vorgeblich uneingeschränkten Führerprinzip. Doch die Freiheit der absoluten Führung auf Reichsebene ahmten nur allzu gern Gauleiter und Ministerpräsidenten in ihren Bereichen nach und waren meist auf eine umfangreiche Machtkompetenz bedacht. Dies spiegelt sich im „Gesetz über den Neuaufbau des Reichs“ vom 30. Januar 1934 wider, das die Hoheitsrechte der Länder dem Reich übertrug; die Länder aber mangels Durchsetzungsmöglichkeiten des Reichs dessen Umsetzung wieder rückübertragen bekamen. Auch das zweite Reichsstatthaltergesetz vom 30. Januar 1935 löste den Grundkonflikt zwischen Zentralismus und Partikularinteressen, anfangs der Länder, dann zunehmend der Reichsstatthalter beziehungsweise Gauleiter, in den zwölf Jahren nationalsozialistischen Regimes nie. Der sächsische Gauleiter Martin Mutschmann, der die Ämter Reichsstatthalter, Ministerpräsident beziehungsweise ,Führer der Landesregierung' und Reichsverteidigungskommissar in seiner Person und damit eine Machtfülle vereinigte wie kein anderer Gauleiter im Reich, ist das Beispiel für das Streben nach einem absoluten Herrschaftsanspruch unter dem ,Führer ${ }^{6}$ Adolf Hitler. Es ist heute so, daß das Reichsrecht an der sächsischen Grenze erlischt, schrieb Heinrich Himmler 1944 über Mutschmanns Herrschaft. ${ }^{7}$ Fast jede Verwaltungsebene im NS-Staat besaß ihren eigenen, zumindest in seinem Fachbereich fast unumschränkt leitenden ,Führer'. Hierzu zählten nach dem sächsischen Ministerpräsidenten auch die Minister, die bisweilen gegeneinander den Zuschnitt des eigenen Ministeriums - ergo den eigenen Machtbereich - vergrößern wollten. Diese ,innerbetriebliche' Konkurrenz war gepaart mit dem fast eifersüchtigen Blick nach Berlin zu den Reichsministerien. Als Nationalsozialisten per se für eine starke Reichsgewalt, reklamierten die Minister jedoch für ihre eigenen Ministerien eine möglichst große Selbstständigkeit gegenüber den Direktiven aus Berlin. ${ }^{8}$ In diesem Sinne waren auch die an zentrale Weisungen gebundenen Minister, selbstständig' und vertraten eigene Herrschaftsansätze.

7 Andreas Wagner, Martin Mutschmann, in: Schmeitzner/Wagner, Macht und Ohnmacht (wie Anm. 4), S. 279-308, hier S. 306.

8 Vgl. z. B.: ANDREAS WAgNer, Partei und Staat. Das Verhältnis von NSDAP und innerer Verwaltung im Freistaat Sachsen 1933-1945, in: Clemens Vollnhals (Hg.), Sachsen in der NS-Zeit, Potsdam 2002, S. 41-56, hier S. 50; JÜrgen John/HorST MÖller/ThomaS SCHAARSCHMidT (Hg.), Die NS-Gaue. Regionale Mittelinstanzen im zentralistischen „Führerstaat“, München 2007. 


\section{Arthur Göpfert-Kurzbiografie}

Diese selbstständigen Herrschaftsansätze sollen exemplarisch für das sächsische Volksbildungsministerium untersucht werden, dem seit 1935 kein Minister, sondern lediglich ein kommissarischer Leiter vorstand: Arthur Göpfert. ${ }^{9}$ Hierbei soll keine Biografie dieses ,alten Kämpfers‘ der sächsischen NSDAP vorgestellt werden, sondern auf die Etablierung der begrenzten Ministerialmacht sowie auf das Verhältnis Göpferts zu dem am 1. Mai 1934 eingerichteten Reichsministeriums für Wissenschaft, Erziehung und Volksbildung unter Bernhard Rust eingegangen werden. Durch die Schaffung des Ministeriums war ein Gegensatz zwischen den Reichsanordnungen und dem Beharren der Länder beziehungsweise Gauleiter auf möglichste Eigenständigkeit in der Bildungs- und Hochschulpolitik entstanden, die ihre Kompetenzen nicht schmälern lassen wollten. Die Hochschulen hatten den Ländern unterstanden, bis das neue Reichsministerium auch dafür seine Zuständigkeit erklärte.

Göpfert erscheint trotz der Bedeutung seines Volksbildungsministeriums nur vereinzelt in Publikationen. Meistens wird er nur im Zuge der ,zweiten Machtergreifung' 1935 oder in der sächsischen Hochschulpolitik genannt. ${ }^{10}$ Als Abgeordneter des kurzlebigen nationalsozialistischen sächsischen Landtags 1933 trat er nicht in Erscheinung, ebenso wenig wie als Abgeordneter des Reichstags, dem er die gesamte NS-Zeit angehörte. Einzig Claus-Christian W. Szejnmann nennt ihn als ein Beispiel eines redegewandten Nationalsozialisten der sächsischen NSDAP aus der ,Kampfzeit' ${ }^{6}{ }^{11}$ Zusammenfassend bleibt Göpfert in den Publikationen als Leiter des schon per se wichtigen Ministeriums für Volksbildung dennoch blass.

Dieser Vertraute des Gauleiters gehörte zur ,Plauener Gauclique‘ des Reichsstatthalters Martin Mutschmann, der aus Plauen stammte, wo bereits seit 1922 eine Ortsgruppe der NSDAP bestand. Aus dieser ,Südwestgemeinschaft' der frühen NSDAP-Ortsgruppen rekrutierte Mutschmann nach 1933 und nach 1935 zahlreiche Parteimitglieder, die in der Gau- und Landesverwaltung eingesetzt wurden. Arthur Göpfert wurde am 7. Juni 1902 im vogtländischen Pausa geboren. ${ }^{12}$ Er war daher im Ersten Weltkrieg kein Kriegsteilnehmer (der letzte eingezogene Jahrgang war der von 1900), sondern besuchte bis 1923 das Lehrerseminar in Plauen. Im gleichen Jahr trat er in die NSDAP ein. In Syrau zunächst Ortsgruppenleiter, wurde er anschließend in Glauchau Stadtverordneter und später Stadtrat. Der als guter Redner geltende Göpfert ließ sich zu einem der 6.000 ,Reichsredner' der NSDAP ausbilden, die reichsweit bei Propagandaveranstaltungen reden durften. In der ,Kampfzeit‘, im Jahr 1930, avan-

9 Arthur Göpfert ist nicht zu verwechseln mit dem gleichnamigen Nationalliberalen: Konstantin Hermann, Arthur Göpfert, in: Sächsische Biografie (wie Anm. 3) (Zugriff 23. März 2011).

$10 \mathrm{Vgl}$. Schmeitzner, Wagner, Vollnhals sowie die weiteren Literaturhinweise im Text. Konstantin Hermann, Arthur Göpfert. Zehn Jahre kommissarischer Leiter des Volksbildungsministeriums, in: PiePer/SCHMEITZner/NASER, Braune Karrieren (wie Anm. 6), S. 226-231.

11 Claus-Christian W. Szejnmann, Nazism in Central Germany. The Brownshirts in „Red“ Saxony (Monographs in German History 4), New York/Oxford 1999, S. 64 f., 171.

12 Vgl. Sächsisches Staatsarchiv - Hauptstaatsarchiv Dresden (im Folgenden: HStA Dresden), 11848 NS-Gauverlag, Nr. 36/G 290, Lebenslauf Göpfert; LiLlA, Statisten in Uniform (wie Anm. 1), S. 302. In den anderen biografischen Nachschlagewerken wie dem von Hermann Weiss, Biographisches Lexikon zum Dritten Reich, Frankfurt am Main 1998; oder ERNST KLEE, Das Personenlexikon zum Dritten Reich. Wer war was vor und nach 1945? Frankfurt am Main 22005, wird Göpfert nicht genannt. 
cierte er zum Gauobmann des Nationalsozialistischen Lehrerbundes (NSLB). ${ }^{13}$ Gemeinsam mit Hans Schemm und Fritz Wächtler hatte er den NSLB 1927 in Hof gegründet. ${ }^{14}$ Der NSLB war für die Durchführung der politisch-weltanschaulichen Ausrichtung aller Lebrer im Sinne des Nationalsozialismus verantwortlich. ${ }^{15}$ Rückblickend hieß es 1940 über die sächsischen Verhältnisse: Schemm fand für den Nationalsozialistischen Lebrerbund gerade in Sachsen die beachtlichsten Helfer, so daß seine Lebrerorganisation für Deutschland in Sachsen zuerst zur Blüte kam. Die Arbeit Göpferts und seines sächsischen NSLB wurde von Schemm als vorbildlich charakterisiert. ${ }^{16}$ Der NSLB erreichte nie eine größere Bedeutung in Sachsen, ähnlich wie in den anderen Gauen. Zu groß waren die Differenzen bezüglich der Schulpolitik zwischen Reich, Ländern und Gauen, zumal der NSLB über zahlreiche interne, strukturbedingte Abstimmungsprobleme verfügte. Wegen der aus dem Ruder laufenden Finanzen wurde der Lehrerverband schließlich am 2. Februar 1943 im gesamten Reich kurzerhand stillgelegt. ${ }^{17}$

Göpfert oblag in Sachsen nach 1933 die Aufgabe, diese NSDAP-Organisation zu einer Gemeinschaftsorganisation aller sächsischen Erzieher im Sinne der Reichsleitung des NSLB durch Hans Schemm umzugestalten. Als Gauamtsleiter des Amts für Erziehung stand er noch über der Gauleitung des NSLB. Mit diesen beiden Funktionen vereinigte er die wichtigsten Schaltstellen der sächsischen Pädagogik. Im März 1933 war der Machtkampf zwischen Mutschmann und dem durch den Reichsinnenminister Wilhelm Frick eingesetzten ,Polizeikommissar' und späteren sächsischen Ministerpräsidenten Manfred von Killinger zunächst zugunsten des letzteren entschieden. Im Zuge dieser Machtübernahme der Nationalsozialisten wurde Göpfert zunächst Beigeordneter im Ministerium für Volksbildung, dann Ministerialrat und ,Reichsbevollmächtigter des NSLB' in Sachsen. Killinger behielt die Ministerialbürokratie aus der Weimarer Zeit und holte nur wenige Parteigenossen in die Ministerien. Dies änderte sich jedoch zwei Jahre später. Als von Killinger als Ministerpräsident abgesetzt und durch Mutschmann als ,Führer der Landesregierung' am 28. Februar 1935 ersetzt wurde, setzte dieser weitere Mitstreiter aus seiner, Gauclique in die Ministerien um. ${ }^{18}$ Der Arbeitsminister Georg Schmidt wurde entlassen und anstelle des von Mutschmann gehassten deutschnationalen Ministers für Volksbildung Wilhelm Hartnacke setzte er am 11. März 1935 als kommissarischen ,Leiter des Ministeriums für Volks-

13 Willi Feiten, Der Nationalsozialistische Lehrerbund. Entwicklung und Organisation. Ein Beitrag zum Aufbau und zur Organisationsstruktur des nationalsozialistischen Herrschaftssystems (Studien und Dokumentationen zur deutschen Bildungsgeschichte 19), Basel 1981.

14 Rolf EILERs, Die nationalsozialistische Schulpolitik. Eine Studie zur Funktion der Erziehung im totalitären Staat, Köln/Opladen 1963, S. 128.

15 Organisationshandbuch der NSDAP, München 1937, S. 252.

16 BenedikT Lochmüller, Hans Schemm, Bd. 1, Bayreuth/München 1940, S. 221.

17 EILERS, Schulpolitik (wie Anm. 14), S. 134.

18 AndREAS WaGNeR, Mutschmann gegen von Killinger. Konfliktlinien zwischen Gauleiter und SA-Führer während des Aufstiegs der NSDAP und der „Machtergreifung“ im Freistaat Sachsen, Beucha 2001; Ders., Martin Mutschmann (wie Anm. 7), hier S. 298 f.; DERS, „Machtergreifung“ in Sachsen. NSDAP und staatliche Verwaltung 1930-1935, Köln/ Weimar/Wien 2004, besonders S. 328-331, 378. 
bildung' dessen Intimfeind, den Gauamtsleiter Arthur Göpfert, ein. ${ }^{19}$ Im Gegensatz zu diesem war Hartnacke noch einer moderaten Bildungspolitik verpflichtet gewesen. ${ }^{20}$ Bereits die Ernennung Göpferts war ein Zeichen eigenständiger sächsischer Kultuspolitik Mutschmanns, der die Beamten des Ministeriums nicht in der Lage sah, die Geschäfte ordnungsgemäß zu führen. Das Reichsinnenministerium hatte hingegen die Länder eigentlich ersucht, auf Neuberufungen von Ministern im Hinblick auf eine Reichsreform zu verzichten. ${ }^{21}$ Mit Göpfert übernahm zudem nicht ein Staatssekretär oder anderer Beamter die Führung des Ministeriums, sondern zum wiederholten Male ein Mitglied der sächsischen Gauleitung. Göpfert, der nominell nie Minister war, hatte dieses Amt bis zum 10. April 1943 inne, als Mutschmann alle Ministerien auflöste und diese im Sinne einer absoluten Herrschaft als Abteilungen der Landesregierung unterstellt wurden.22 Die Befugnisse der Abteilungsleiter waren noch deutlicher eingeschränkt als die der früheren Minister. Göpfert blieb bis zum Zusammenbruch im Mai 1945 Leiter der Abteilung Wissenschaft, Erziehung und Volksbildung. Nach Ablehnung der von Mutschmann angebotenen „Unabkömmlichkeit“ diente Göpfert 1939 und ab 1943 in der Luftnachrichtentruppe, wurde 1945 kriegsgefangen und musste bis 1948 im Steinbruch arbeiten. Er musste sich einem Entnazifizierungsverfahren stellen, arbeitete wieder als Lehrer, trat öffentlich nicht mehr in Erscheinung und stand anscheinend lediglich Raimund Baumgärtner als Zeitzeuge zur Verfügung. ${ }^{23}$ In Söchtenau bei Rosenheim in Bayern starb Göpfert am 23. Februar 1986. ${ }^{24}$

Als Leiter des Ministeriums für Volksbildung verantwortete Göpfert zahlreiche Aufgabengebiete: Schule, Hochschulen, die Staatlichen Sammlungen für Kunst und Wissenschaft, Stiftungen und Kirchen. 1936 wies der Geschäftsverteilungsplan des Ministeriums zwölf Referate auf. ${ }^{25}$ Auf Göpferts Rolle bei den Staatlichen Sammlungen für Kunst und Wissenschaft soll zugunsten einer Schwerpunktsetzung dieses Artikels auf Bildung und Wissenschaft sowie Volkskunde nicht vertieft eingegangen

19 Dresdner Nachrichten, 23. März 1935. Im März 1935 vereinigte Mutschmann auch die Staatskanzlei mit der Statthalterei. Übel genug, doch ein gebildeter Mann, mehr rechts als Nazi schrieb Victor Klemperer über Hartnacke. VICTOR KlEMPERER, Ich will Zeugnis ablegen bis zum Letzten. Tagebücher 1933-1941, hrsg. von Walter Nowojski, Berlin 1995, S. 189 f. Klemperer gibt die Meinung eines Bekannten über Göpfert wieder, der ihn als Idealisten, nicht bösartig, aber mit viel Rancune des kleinen Mannes bezeichnet hatte; ebd., S. 192. Mutschmann schätzte Göpfert als zu nachgiebig ein, und das Verhältnis beider gestaltete sich zunehmend schwieriger.

20 Thomas SchaARSCHMidT, Regionalkultur und Diktatur. Sächsische Heimatbewegung und Heimat-Propaganda im Dritten Reich und in der SBZ/DDR, Köln/Weimar 2001, S. 39 .

21 Michael Parak, Hochschule und Wissenschaft in zwei deutschen Diktaturen. Elitenaustausch an sächsischen Hochschulen 1933-1952 (Geschichte und Politik in Sachsen 23), Köln/Weimar/Wien 2004, S. 86.

22 Geschäftsverteilungsplan vom 1. April 1943, HStA Dresden, 10851 Ministerium der Finanzen, Nr. 13251, unpag. Zum Etat des Ministeriums 1935 bis 1942 siehe HStA Dresden, 11125 Ministerium des Kultus und öffentlichen Unterrichts, Nr. 10408/37-41.

23 Raimund BAumgäRTNER, Weltanschauungskampf im Dritten Reich. Die Auseinandersetzung der Kirchen mit Alfred Rosenberg (Veröffentlichungen der Kommission für Zeitgeschichte B/22), Mainz 1977, S. XI.

24 Auskunft der Gemeinde Söchtenau durch Elisabeth Gnadl vom 7. April 2011.

25 HStA Dresden, 10851 Ministerium der Finanzen, Nr. 13248, unpag. 
werden. ${ }^{26}$ Auf die sächsische Kirchenpolitik nahm Göpfert nur geringen Einfluss, weshalb sie nur angerissen werden soll. ${ }^{27}$

Göpfert war zwar Mitglied der sächsischen Landessynode, trat aber dort nicht in Erscheinung. Er hielt sich an die Reichsregelung, sich in innerkirchliche Angelegenheiten nicht einzumischen. Im Falle der durch die Deutschen Christen geforderten Abberufung des Pfarrers Hänichen in Hohenfichte wertete der Ministeriumsleiter dies gegenüber dem deutsch-christlichen Landeskirchenamt als innerkirchliche Angelegenheit und lehnte eine Intervention ab. Göpfert berief sich sogar auf die Reichsverfassung von 1919, die den Religionsgemeinschaften freie Wahl bei ihren Beamten zusicherte. Nur wenn der Pfarrer staatsfeindlich aktiv sei, würde Göpfert dies der Gestapo mitteilen. ${ }^{28}$ Der sächsische Landesbischof schrieb an Göpfert, dass die Trennung von Staat und Kirche in der marxistischen Zeit entstanden sei, und meinte damit die Weimarer Reichsverfassung von 1919.29 Dadurch, so Coch, würde es der Exekutive erschwert, gegen missliebige Pfarrer vorzugehen. Auch in der Kirchenpolitik fanden in der NSZeit die typischen Auseinandersetzungen zwischen Reichsgewalt und Länderinteressen statt. Die Finanzabteilung des Landeskirchenamts berichtete direkt an das Reichskirchenministerium, was Mutschmann 1936 bewog, Göpfert in Gesprächen mit der Finanzabteilung bewusst zu machen, dass diese an das vorgesetzte Ministerium, nämlich das Volksbildungsministerium, zu berichten habe. ${ }^{30}$ Dies wurde auch dem Reichskirchenminister mitgeteilt.

26 Vgl. dazu: Birgit SchWARZ, Geniewahn. Hitler und die Kunst, Köln/Weimar/Wien 2009 (vor allem zur Absetzung von Hans Posse und Göpferts Rolle dabei); KONSTANTIN HeRMANN, Die Sächsische Landesbibliothek in der nationalsozialistischen Zeit, in: NASG 80 (2009), S. 277-290 (hier auch Göpferts Wirken auf die Bibliothekspolitik); Ders., Die Sächsische Landesbibliothek 1933-1945. Martin Bollert und Hermann Neubert - Zwei Epochen in zwölf Jahren?, in: Michael Knoche (Hg.), Wissenschaftliche Bibliothekare im Nationalsozialismus. Handlungsspielräume, Kontinuitäten, Deutungsmuster, Wiesbaden 2011, S. 289-308.

27 Vgl. z. B. Birgit Mitzscherlich, Diktatur und Diaspora. Das Bistum Meißen 1932-1951 (Veröffentlichungen der Kommission für Zeitgeschichte B/101), Paderborn u. a. 2005; KARL-JOSEPH Hummel/CHRISTOPH KÖsTERS, Zwangsarbeit und katholische Kirche 1939-1945. Geschichte und Erinnerung, Entschädigung und Versöhnung. Eine Dokumentation (Veröffentlichungen der Kommission für Zeitgeschichte. Reihe B/110), Paderborn u. a. 2008; GeORG WilHelm, Die Diktaturen und die evangelische Kirche. Totaler Machtanspruch und kirchliche Antwort am Beispiel Leipzigs 1933-1958 (Arbeiten zur kirchlichen Zeitgeschichte. Reihe B/39), Göttingen 2004. Vgl. allgemein HStA Dresden, 11125 Ministerium des Kultus und öffentlichen Unterrichts, Nr. 13058/175 Deutsche Evangelische Kirche 1933-1937, Nr. 13058/5-6 Kirchenaustrittsgesetz.

28 HStA Dresden, 11125 Ministerium des Kultus und öffentlichen Unterrichts, Nr. 13058/40 Neuordnung des Verhältnisses der ev.-luth. Landeskirche Sachsen nach 1933, fol. 45-52 (Schreiben Göpferts vom 26. April 1935). Vgl. allgemein: JOACHIM FISCHER, Die sächsische Landeskirche im Kirchenkampf 1933-1937, Göttingen 1972. Göpfert erscheint in den Monografien und in den Erinnerungen der Protagonisten des Kirchenkampfes nur selten.

29 HStA Dresden, 11125 Ministerium des Kultus und öffentlichen Unterrichts, Nr. 13058/40, fol. 91 (Schreiben Coch an Göpfert vom 18. Juni 1935).

30 HStA Dresden, 11125 Ministerium des Kultus und öffentlichen Unterrichts, Nr. 13058/77, fol. 96 (Aktennotiz). Vgl. auch: FISCHER, Landeskirche im Kirchenkampf (wie Anm. 28), S. 165. 


\section{II. „Rasse, Volk, Wehr, Fübrertum, Religiosität“ - Göpferts Schulpolitik}

Wie in vielen anderen Bereichen der Regierungspolitik verfügte die NSDAP auch in der Schul- und Wissenschaftspolitik kaum über Programme, die über Kernthesen hinausgingen. Der durch Volksschullehrer wie Schemm und Göpfert geprägte NSLB verlangte vor allem die Auflösung der Lehrervereine, die Einführung der Gemeinschaftsschule und die akademische Ausbildung der Volksschullehrer. Der NSLB wurde durch Schemm nach 1933 von einem „politischen Agitationsverband“ in eine Pädagogenorganisation umgewandelt, die 1937 schließlich 97 Prozent aller deutschen Lehrer zu seinen Mitgliedern zählte. ${ }^{31}$ Die Ernennung eines NSLB-Funktionärs zum Kultus- beziehungsweise Volksbildungsminister nach der ,Machtergreifung waren seltene Ausnahmen; Göpfert zählte dazu. Wie beim NSLB im Reich lag das Hauptinteressengebiet auch des sächsischen NSLB unter Göpfert auf den Mittel- und Volksschulen. ${ }^{32}$

Göpferts Auffassungen von Pädagogik, die die Grundlagen seiner Ministeriumsarbeit waren, lassen sich am besten durch seine Publikationen belegen. „Bildung und Arbeit" bildeten den Schwerpunkt seiner Arbeit. Arthur Göpfert hatte bereits 1932 im Auftrag des sächsischen NSLB das Buch „Nationalsozialistische Lehrerbibliothek“ herausgegeben. Im Juli 1933 erschien das erste Heft der bis September 1939 gedruckten Monatszeitschrift des NSLB Sachsen „Politische Erziehung“, die durch Göpfert herausgegeben wurde. ${ }^{33} \mathrm{Im}$ äußerst programmatischen Geleitwort zum ersten Heft versuchte der Herausgeber, das Wort ,Pädagogik' zu ersetzen, die in den nächsten Jabrhunderten ,politische Erziebung' genannt werden sollte. ${ }^{34}$ Der Sinn der modernen Staatsidee sei erfüllt, wenn Adolf Hitler, der Hingabebereiteste und Dienstwilligste vor allen anderen, eine fast absolute Machtvollkommenheit besitzt. Der Lehrer, einst nur Pädagoge, sei künftig politisch, sein Leben [sei] eine Nachfolge des Kanzlers. Die Erziehungsziele des NS-Staates seien Rasse, Volk, Wehr, Führertum und Religiosität. Die mit diesen pathetischen Worten eingeleitete Zeitschrift vereinigte Artikel von Kurt Arnold Findeisen, Guido Erwin Kolbenheyer, Wolfgang Willrich und anderen. Göpfert gab auch die „Bausteine für die deutsche Erziehung. Dürr’s methodische Heftereihe“ (1934 bis 1942) heraus, die anders als die „Politische Erziehung“ vor allem fachpädagogische und methodische Beiträge brachte. 1933/34 fungierte er als Herausgeber von „Dürr's vaterländischen Bücherei“, die nur kurz erschien und deren zweites Heft der Biografie Adolf Hitlers gewidmet war. In dem von Göpfert herausgegebenen zweibändigen Werk „Denkmal deutscher Arbeit“ fanden sich Artikel zahlreicher Grö-

31 EILERS, Schulpolitik (wie Anm. 14), S. 128.

32 HStA Dresden, 11125 Ministerium des Kultus und öffentlichen Unterrichts, Nr. 14620 NSLB.

33 Vgl. auch: MatThias Middell, Weltgeschichtsschreibung im Zeitalter der Verfachlichung und Professionalisierung. Das Leipziger Institut für Kultur- und Universalgeschichte 1890-1990 (Geschichtswissenschaft und Geschichtskultur im 20. Jahrhundert 6), Leipzig 2005, S. 658.

34 ARTHUR GÖPfERT, Politische Erziehung, in: Politische Erziehung 1 (1933), S. 1. Zu Göpfert hinsichtlich des Leipziger jüdischen Schulwerks siehe: BARBARA KOWALZIK, Lehrerbuch. Die Lehrerinnen und Lehrer des Leipziger jüdischen Schulwerks 1912-1942 (Leipziger Kalender Sonderband 2006/1), Leipzig 2006. Der NSLB Sachsen gab auch die Monatszeitschrift „Das politische Semester“ heraus, deren Chefredakteur Göpfert war. 
ßen der NS-Prominenz wie Alfred Rosenberg, Baldur von Schirach und Robert Ley. ${ }^{35}$ Die Fachartikel sind dagegen durchaus informativ.

Wie andere Ministerien war auch das Ministerium für Volksbildung von 1933 bis 1935 dem direkten Einfluss der sächsischen NSDAP entzogen. Der Gauamtsleiter Göpfert konnte nur über seinen NSLB Einfluss nehmen. Mit Hartnacke stand dem Nationalsozialisten zudem noch ein Deutschnationaler gegenüber, der auf die Richtlinienkompetenz seines Ministeriums achtete. Dem NSLB blieb nur, direkt oder indirekt seine Vorstellungen von Pädagogik im Ministerium für Volksbildung durchzusetzen, was ihm zunehmend gelang. Die erwähnte Göpfertsche Vorstellung von Pädagogik, nämlich Rasse, Volk, Wehr, Führertum und Religiosität, spiegelte sich in seinen Ministeriumsanordnungen erkennbar wider und führte zu einer deutlichen Politisierung der Schulen. Auf Anregung des NSLB wurde an den Schulen aller Arten 1934 ,Schuljugendwalterämter' für Lehrer eingerichtet. Die Lehrer, die diese Ämter innehatten, mussten die Verbindung mit der NSDAP und ihren Organisationen wie dem sozialen Amt der HJ, der Deutschen Arbeitsfront, aber auch zu Jugendherbergen u. a. halten, die für die älteren Schüler relevant waren. ${ }^{36}$ Die Schulen wurden für Werbeaktionen der HJ genutzt, die Göpfert anordnete. Legion waren reichsweit die Beschwerden über den ausufernden HJ-Dienst, der auch in Sachsen zu mehreren Verordnungen Göpferts führte. ${ }^{37}$ Der Ministeriumsleiter ermahnte die Schulbezirke, darauf zu achten, dass alle Kräfte der Schüler der Schule zukommen müssten und die HJ die erforderliche Rücksicht auf die Schule aufbringen müsse. Auch den Schüleraustausch passte Göpfert der NS-Ideologie an. Im Vordergrund standen nun die nordischen Länder, wohin die höheren Schüler reisen konnten. Vor 1933 war der Schüleraustausch mit diesen Ländern zurückgegangen. ${ }^{38}$ In Dresden wurde der deutsch-schwedische Schüleraustausch organisiert, der proportional der größte Schüleraustausch in Deutschland war. Erzieherisch wirkt das Kennenlernen eines artverwandten Volkes [...]. Der charakterbildende Wert liegt darin [...], daß die Jungen und Mädchen einmal auf sich selbst gestellt sind und sich als Deutsche zu bewähren haben. ${ }^{39}$

Die Lehrer wurden aufgefordert, an Lehrgängen für Rassenkunde und Rassenpflege teilzunehmen und Luftschutzunterricht zu erteilen. Fuß- und Handball wurden als ,Kampfspiele' gefördert, die mit allen Schülern ab dem 7. Schuljahr zu betreiben waren. ${ }^{40}$ Auch die Erstellung von Ahnen- und Sippschaftstafeln wurde vom Volksbildungsministerium propagiert. Göpfert verpflichtete am 21. Juni 1935 alle Lehrer und Schüler, auch außerhalb der Schulen, zur Verwendung des Hitlergrußes ${ }^{41}$ und gab im Dezember 1935 neue Vorschriften für Schulleiter und Lehrer an Volks-, Hilfs- und Berufsschulen aus, die nunmehr Erzieber zur nationalsozialistischen Staatsgesinnung und Gestalter der deutschen Zukunft sein sollten. Die Schulleiter waren angehalten, die Schulen nach dem Willen des Fübrers unter alleiniger Verantwortung zu leiten. ${ }^{42}$

35 ARThur GöPfert (Hg.), Denkmal deutscher Arbeit. Ein Werk von deutscher Kraft und Tüchtigkeit für Jugend, Schule, Arbeitsdienst, Gewerbe, Industrie und Handel zur Selbstunterrichtung, 2. Bde., Leipzig 1934/35.

36 Verordnungsblatt des Ministeriums für Volksbildung (im Folgenden: Verordnungsblatt) 16 (1934), Nr. 11, S. 53.

37 Zum Beispiel: Verordnungsblatt 21 (1939), Nr. 1, S. 11 f.

38 Verordnungsblatt 16 (1934), Nr. 9, S. 41.

39 Verordnungsblatt 20 (1938), Nr. 7, S. 36 f.

40 Verordnungsblatt 18 (1936), Nr. 4, S. 12.

41 Verordnungsblatt 17 (1935), Nr. 14, S. 69.

42 Verordnungsblatt 17 (1935), Nr. 25, S. 149 f. 
Nach der Anweisung des Reichserziehungsministeriums vom 2. Juli 1937 zu den Auswirkungen des Reichsbürgergesetzes verlangte das sächsische Volksbildungsministerium von den Schulbezirken mit möglichster Beschleunigung zu untersuchen, ob und in welcher Weise die jüdischen Schüler abgesondert werden könnten. Der hebräische Sprachunterricht an den höheren Schulen wurde zum 1. Dezember 1937 in Sachsen eingestellt, für jüdischen Religionsunterricht durften keine Schulräume mehr genutzt werden. ${ }^{43}$ Die Behandlung des Judentums im Religionsunterricht wurde 1938 durch Göpfert verboten, jedoch sollte die Schule bei ihren Schülern das Bewusstsein von der Schädlichkeit der jüdischen Rasse und von ibrem Hasse gegen alles Deutsche schaffen und wach halten. ${ }^{44}$ Werbeaushänge für kirchliche Veranstaltungen durften in Schulen seit dem 5. März 1938 durch eine Verordnung Göpferts nicht mehr angebracht werden. ${ }^{45}$ Auch die sorbischen Schulen gerieten durch die Germanisierungsabsichten der Nationalsozialisten und so auch durch Göpferts Wirken als Ministeriumsleiter unter Druck. ${ }^{46}$

Den einzelnen Fachbereichen innerhalb des NSLB, auf die die Lehrer nach den unterrichteten Fächern aufgeteilt waren, kam nach der Ernennung Göpferts zum Ministeriumsleiter auch die Rolle der Indoktrination zu. Besonders traf dies auf die historisch-geografischen und biologischen Fächer zu. Auf dem Ersten Sächsischen Schulgeographentag in Dönschten bei Dippoldiswalde vom 1. bis 3. Juni 1935 behandelte das erste Referat Sachsen als Teil der deutschen Ostfront - mit dem vorweggenommenen Vokabular von 1941.47 Sachsens Funktion als Grenzland, als ein ,Vorposten gegen das benachbarte Slawentum' wurde von den Nationalsozialisten gern apostrophiert. Der Referent beklagte jedoch dabei den grenzländischen Wirtschaftsegoismus der Erzgebirger, die gern in der Tschechoslowakei wegen der günstigeren Preise einkauften. Die Göpfertsche Neuordnung des Büchereiwesens an Volksschulen unterstellte alle Schulbibliotheken der strengen Aufsicht der Landesfachstelle für Schülerbüchereien. Am 22. April 1938 gab Göpfert eine genaue Bücherliste vor, von der Abweichungen nur im genehmigten Einzelfall zulässig waren. Diese Liste zu erwerbender Bücher umfasste zu zwei Dritteln Literatur zur germanischen, deutschen, Weltkriegs- und Kolonialgeschichte sowie zur ,Kampfzeit' der NSDAP und zum ,neuen Reich'. ${ }^{48}$ Das 1939 erschienene neuartige Bildkartenwerk für sächsische Schulen „Seht, das ist Sachsen!“, das Mutschmann und Göpfert durch ihre Geleitwörter ,adelten', sollte die Einsatzbereitschaft für das große Vaterland hervorrufen. Neben Bildkarten über Siedlungsformen, Handelsstraßen und bedeutende Sachsen fanden

43 Verordnungsblatt 19 (1937), Nr. 21, S. 126 f.

44 Verordnungsblatt 20 (1938), Nr. 24, S. 135.

45 Verordnungsblatt 20 (1938), Nr. 6, S. 31.

46 Vgl. auch: FrAnK FÖRSTER, „Lebensraumkundlich“ zur „Wendenfrage“. Wege und Stationen eines Arbeitsgemeinschaftsleiter des Nationalsozialistischen Lehrer-Bundes im Kreis Löbau, in: Lětopis 48 (2001), Nr. 2, S. 29-37; EDMUND PECH, Auswirkungen des Nationalsozialismus auf die Volksschulen in den „wendischen und gemischtsprachigen Gebieten“" in Sachsen, in: Lětopis 50 (2003), Nr. 2, S. 3-21; DERS., Auswirkungen des Nationalsozialismus auf die zweisprachigen katholischen und evangelischen Kirchgemeinden in der Oberlausitz, in: Lars-Arne Dannenberg (Hg.), Stätten und Stationen religiösen Wirkens. Studien zur Kirchengeschichte der zweisprachigen Oberlausitz (Schriften des Sorbischen Instituts 48), Bautzen 2009, S. 314-323.

47 HStA Dresden, 11125 Ministerium des Kultus und öffentlichen Unterrichts, Nr. 14620 NSLB Erdkunde-Volkskunde 1935-1939, fol. 12.

48 Verordnungsblatt 20 (1938), Nr. 10, S. 56-59. 
sich auch die kommentierten Karten „Straßen des Führers“, „Sachsen als Stoßtrupp in der Bewegung“ und „Vom Grenzland zum Herzen Großdeutschlands“.49

In der Schulpolitik versuchte die sächsische Regierung sich gegen den Berliner Zentralismus durchzusetzen. Göpfert hatte sich 1937 beim Reichserziehungsministerium beklagt, dass in Sachsen noch mit dem Landeslehrplan mit dem liberalistischen Geist von 1928 gearbeitet würde und kündigte die Einführung von zwei durch den NSLB erarbeiteten Volksschullehrplänen an, die die Zeit bis zu den geplanten Reichsrichtlinien überbrücken sollten. ${ }^{50}$ Das Erziehungsministerium forderte Göpfert, der schon zum 6. April 1937 die neuen Lehrpläne verbindlich eingeführt hatte, zu spät zum Verzicht auf. Die einige Tage später durch Rust erlassenen Reichsrichtlinien erlangten in Sachsen aufgrund des Widerstands von Göpfert keine Gültigkeit. Die beiden sächsischen Lehrpläne sollten die Kinder zum Dienst für den nationalsozialistischen Staat erzieben. ${ }^{51}$ Nicht nur bei sachsenweiten Bildungsthemen, sondern auch bei regionalen beziehungsweise lokalen, versuchte Göpfert den Anspruch des eigenen Ministeriums gegenüber der Reichsbehörde zu wahren.

Die Bewahrung einer eigenständigen Bildungspolitik zeigte sich 1935 zum Beispiel an der Scharnhorstschule in Dresden-Striesen - dem ehemaligen Freimaurerinstitut und deren in Schwierigkeiten gekommenen Träger. Göpfert verhandelte mit dem Stiftungsrat über eine Anerkennung als Stiftung öffentlichen Rechts und die Übernahme von Ausgaben durch das Land Sachsen. Das Land trug bereits sämtliche Personalkosten des Lehrkörpers. Schon im Mai 1935 hatte Göpfert auf das ungünstige Verhältnis von planmäßig angestellten Lehrern und Studienassessoren an der Scharnhorstschule hingewiesen. Favorisiert hatte die Stiftung privaten Rechts zunächst die Umwandlung in eine Deutsche Heimschule, die dann dem Reichserziehungsministerium unterstanden hätte. ${ }^{52}$ Die auf Befehl Hitlers gegründeten Heimschulen waren Teil der Eliteschulausbildung der Nationalsozialisten und sollten vor allem Kindern von Soldaten oder Gefallenen eine Ausbildung mit unterschiedlichen Abschlüssen bieten. Göpfert stellte dem Stiftungsträger gegenüber jedoch klar, dass die finanziellen Zuwendungen des Landes nach dem Krieg wegfallen würden. ${ }^{53}$ Eine Stiftung privaten Rechts wäre dann durch den von den Nationalsozialisten betriebenen Abbau von Privatschulen kaum zukunftsfähig geblieben, sodass Göpfert mitteilte, die Auflagen des Reichserziehungsministeriums für eine Privatschule seien zu schwierig, und anregte, dass das Land Sachsen die Schule übernimmt. Der Stiftungsrat stimmte dem zu und so kam die Schule nebst lohnenswerten Aktiva von 1.367.133 Reichsmark und kaum vorhandenen Passiva am 1. April 1943 unter die Hoheit des Volksbildungsministeriums. Somit war die Scharnhorstschule dem Zugriff des Reichserziehungsministeriums entzogen.

Seit Februar 1936 gab das Verordnungsblatt des Ministeriums vor allem die Erlasse des Reichserziehungsministeriums wider, nannte Beförderungen und Gehaltsverordnungen sowie ab 1939 kriegsbedingte Maßnahmen wie der Zusammenarbeit von NS-

49 EgOn RenNert/Kurt EgGert, Seht, das ist Sachsen! Ein volkstümliches Bildkartenwerk, Dresden 1939. Eine Einleitung schrieb der Geschäftsführer des Heimatwerks Sachsen, Arthur Graefe. Es erschien im Kommunalverlag Dresden.

50 Margarete GötZ, Die Grundschule in der Zeit des Nationalsozialismus. Eine Untersuchung der inneren Ausgestaltung der vier unteren Jahrgänge der Volksschule auf der Grundlage amtlicher Maßnahmen, Bad Heilbrunn 1997, S. 267-276, hier S. 267.

51 Erziehungs- und Unterrichtsplan für die sächsische Landschule, Dresden 1936, S. 72.

52 Walter Ruge, Die deutschen Heimschulen, Berlin 1942.

53 HStA Dresden, 11125 Ministerium des Kultus und öffentlichen Unterrichts, Nr. 11864/484 Deutsche Heimschule/Scharnhorstschule, fol. 18 (Schreiben Göpfert an den Vorsitzenden der Stiftung, Jensen, vom 10. Oktober 1942). 
Fliegerkorps und Schulen, um zukünftige Piloten zu gewinnen. ${ }^{54} 1939$ führte das Ministerium lediglich eingeschränkt die durch das Reichserziehungsministerium geforderte Einführung der Mittelschulen in Sachsen durch und stellte dies den Schulbezirken frei. ${ }^{55}$ Göpfert legte besonderes Augenmerk auf die bisweilen stark benachteiligten Landschulen. Nur wenige Verordnungen hatten Bedeutung wie der im Schuljahr 1942/43 eingeführte Schultyp der Hauptschule; die meisten Reformmaßnahmen sollten der Nachkriegszeit nach dem ,Endsieg' vorbehalten sein. Dies galt jedoch auch für das Reichserziehungsministerium und andere Reichsministerien.

Zentralisierung oder Regionalisierung bildete sich nicht nur im Verhältnis des Reichs zu den Ländern ab, sondern auch innerhalb der Länder und Gaue selbst. Die sächsische Pädagogenausbildung für das höhere Lehramt wurde am Institut für praktische Pädagogik an der Universität Leipzig konzentriert, um die Auslese einer einzigen Stelle zuzuweisen; das pädagogische Seminar an der TH Dresden wurde 1935 geschlossen. ${ }^{56}$ Göpfert unterlief zudem den Erlass des Reichserziehungsministeriums vom 30. Mai 1941, der bei den Prüfungen für die Studenten des höheren Lehramts Änderungen vorsah, da in Sachsen die in diesem Erlass geschilderte Zweiteilung der Prüfung so nicht bestand. ${ }^{57}$

\section{Eigenständige sächsische Wissenschaftspolitik und das Reichserziebungsministerium}

Auch für den Hochschulbereich verfügten die Nationalsozialisten kaum über ein tragfähiges Entwicklungsprogramm. Grundlage war hier, was Hans Schemm 1933 in München favorisierte, dass es nicht darauf ankäme, ob etwas wabr ist, sondern ob es im Sinne der nationalsozialistischen Revolution sei. ${ }^{58}$ Zunächst wurde in den Hochschulen das Führerprinzip durchgesetzt und damit die traditionelle Selbstständigkeit der Universitäten abgeschafft; missliebige Lehrkräfte wurden aus dem Dienst entfernt. Hartnacke hatte den unter Angriffen Göpferts leidenden Pädagogikprofessor Theodor Litt gedeckt, dem der Gauobmann vorwarf, durch den Kampf gegen die nationalsozialistische Studentenschaft [...] stark belastet zu sein.59 Dem erst 1935 eingesetzten Rektor der Universität Leipzig, Felix Krueger, sagte Göpfert reaktionäre Gesinnung

54 Verordnungsblatt 23 (1941), Nr. 17, S. 86.

55 Verordnungsblatt 21 (1939), Nr. 4, S. 13 f.

56 Verordnungsblatt 16 (1934), Nr. 11, S. 51.1936 wurde dafür die Hochschule für Lehrerbildung gegründet.

57 HStA Dresden, 11125 Ministerium des Kultus und öffentlichen Unterrichts, Nr. 11864, fol. 204 (Schreiben Göpfert an Reichserziehungsministerium vom 12. August1941).

58 UlRICH VON HeHL u. a. (Hg.), Geschichte der Universität Leipzig 1409-2009, Bd. 3: Das zwanzigste Jahrhundert 1909-2009, Leipzig 2010, S. 182; Helmut HeIBER, Universität unterm Hakenkreuz, 3 Bde., München 1991-1994.

59 CARsten Heinze, Die Pädagogik an der Universität Leipzig in der Zeit des Nationalsozialismus 1933-1945. Zur regionalen „Verfallsgeschichte“ einer Wissenschaftsdisziplin, in: Ulrich von Hehl (Hg.), Sachsens Landesuniversität in Monarchie, Republik und Diktatur. Beiträge zur Geschichte der Universität Leipzig vom Kaiserreich bis zur Auflösung des Landes Sachsen 1952, Leipzig 2005, S. 385-410, hier S. 392; WOLFGANG KLAFKI, Die Pädagogik Theodor Litts. Eine kritische Vergegenwärtigung, Königstein/Taunus 1982; WolFGANG SCHWIEDRZIK, Lieber will ich Steine klopfen. Der Philosoph und Pädagoge Theodor Litt in Leipzig (1933-1947), Leipzig 1996, S. 21; Gerald Wiemers, Trennendes und Verbindendes. Zum Dialog zwischen dem Geisteswissenschaftler Theodor Litt und dem Naturwissenschaftler Werner Heisenberg, in: Landesgeschichte und Archivwesen. Fest- 
nach, sodass dieser noch im gleichen Jahr durch den neuen Ministeriumsleiter beurlaubt wurde. ${ }^{60}$ In demselben Jahr sandte Göpfert auch dem Romanisten Victor Klemperer einen Brief, in dem er ankündigte, ihn aufgrund des Gesetzes zur Wiederherstellung des Berufsbeamtentums zur Entlassung vorzuschlagen. ${ }^{61}$

Die deutschen Hochschullehrer, die zum großen Teil dem Nationalsozialismus kritisch oder teilnahmslos gegenüberstanden beziehungsweise eine überparteiliche Wissenschaftlichkeit für sich reklamierten, sollten für Hitler gewonnen werden, zumal am 12. November 1933 die Volksabstimmung über den Austritt Deutschlands aus dem Völkerbund anstand. Göpfert organisierte dazu eine „Treue-Veranstaltung“ in der Alberthalle Leipzig und leitete als Gauobmann des NSLB auch am 11. November das „Bekenntnis der Professoren an den deutschen Universitäten und Hochschulen zu Adolf Hitler und dem nationalsozialistischen Staat" durch eine Rede ein. ${ }^{62}$ Dies geschah noch im Spannungsfeld, als Volksbildungsminister Hartnacke noch amtierte und Göpfert lediglich durch seine Parteiämter Akzente setzen konnte. Hartnacke stand dabei jedoch zunehmend unter dem Druck von Gauleitung, Ministerpräsidenten und auch Göpfert, zumal am 1. Februar 1934 der stramme Nationalsozialist und Gauschulungsleiter Werner Studentkowski als Oberregierungsrat im Volksbildungsministerium eingestellt wurde und später auch als Referent für die sächsischen Hochschulen zuständig war. Er wurde ausdrücklich für die Verflechtung von NSDAP und Gauleitung im Ministerium angestellt. Wie Göpfert gehörte Studentkowski, als im Jahr 1903 Geborener, zur jüngeren Generation der sächsischen Führungselite der NSDAP.

Auch in der Hochschullandschaft kam es zum im NS-Regime typischen Kompetenzwirrwarr. An der Technischen Hochschule Dresden sowie an der Universität Leipzig kam es zu zahlreichen Auseinandersetzungen zwischen Hochschulleitung, Volksbildungsministerium und Reichserziehungsministerium, insbesondere über die Personalpolitik, die das Reich verantworten wollte. ${ }^{63}$ Mutschmann verwahrte sich gegen diesen Berliner Zentralismus. Vor allem dem Leiter des Volksbildungsministeriums gelang es gegenüber den Ambitionen Rusts, der eine Zentralverwaltung der Universitäten und Hochschulen durchsetzen wollte, entgegenzutreten und sogar die Eigenständigkeit der sächsischen Hochschulen zu stärken. ${ }^{4}$ Ganz im Mutschmannschen Sinne hatte Göpfert 1940 beim Rektorwechsel an der Universität Leipzig erklärt,

schrift für Reiner Groß zum 65. Geburtstag, Dresden 2002, S. 455-464; AlberT Reble, Theodor Litt. Eine einführende Werkschau, Bad Heilbrunn 1996, S. 184.

60 Von HeHL, Geschichte der Universität Leipzig (wie Anm. 58), S. 214.

61 Klemperen, Tagebücher (wie Anm. 19), S. 195 f.

62 Cornelia Wegeler, „... wir sagen ab der internationalen Gelehrtenrepublik“. Altertumswissenschaft und Nationalsozialismus. Das Göttinger Institut für Altertumskunde 1921-1962, Köln/Weimar/Wien 1996, S. 229; LeONORE SIEGELE-WENSCHKEWITZ/ Carsten Nicolaisen, Theologische Fakultäten im Nationalsozialismus, Göttingen 1993; VICTOR FARIAS, Heidegger und der Nationalsozialismus, Frankfurt am Main 1989, S. 219225; vON HeHL, Geschichte der Universität Leipzig (wie Anm. 58), S. 208. Der ,Topos der Überparteilichkeit' von Hochschullehrern stand nicht unbedingt im Widerspruch zur Mitgliedschaft in Parteien, so auch der NSDAP. An einigen Universitäten gehörten ca. 25 \% der deutschen Professoren in der Weimarer Republik einer Partei an - oft wird aber die ,Überparteilichkeit der Wissenschaft' auch von ihnen zumindest postuliert worden sein.

63 Vgl. dazu: Reiner Pommerin, Geschichte der TU Dresden 1828-2003, Köln/ Weimar/Wien 2003; vON HEHL, Geschichte der Universität Leipzig (wie Anm. 58).

64 Vgl. auch: Michael PARAK, Hochschule und Wissenschaft. Nationalsozialistische Hochschul- und Wissenschaftspolitik in Sachsen 1933-1945; in: Vollnhals, Sachsen in der NS-Zeit (wie Anm. 8), S. 118-132, hier S. 128. 
dass die Hochschule als Einrichtung [...] gaueigen ist. [...] Vom jüngsten Studenten bis hinauf zum Rektor ist jeder in seiner Haltung dem Gauleiter verantwortlich [...]. ${ }^{65}$

Unter Verschiebung des Schwerpunktes der sächsischen Forschungslandschaft in Richtung Natur- und Technikwissenschaften blieb unter Göpfert der Zustand der Hochschullandschaft bis 1945 relativ konstant. ${ }^{66}$ Ihm gelang es auch, die sächsischen Hochschulen vor einem durch das Finanzministerium geplanten weitreichenden Personalabbau zu bewahren. ${ }^{67}$ Göpfert zog in den zehn Jahren seiner Amtszeit fast alle Unterrichts- und wissenschaftlichen Lehranstalten in den Verantwortungsbereich seines Ministeriums, sodass bereits vor dem Zweiten Weltkrieg sein Bereich nicht nur alle Schulen und wissenschaftlichen Einrichtungen, sondern das gesamte sächsische Bildungswesen umfasste. 1936 gelangte so die Bergakademie Freiberg, 1937 die Kunstgewerbeakademie, die sächsischen Staatsbauschulen und zahlreiche andere staatliche und nichtstaatliche Lehranstalten wie auch Klöppelschulen in die Verantwortung des Göpfertschen Ministeriums, 1939 folgte noch das Kunstgewerbemuseum. ${ }^{68}$

\section{Brauchtumspflege und Volkskunde}

Schon im Juli 1933 richtete Göpfert im NSLB Sachsen die „Landesstelle für Volksforschung und Volkstumspflege“ ein, die beispielgebend für ein inhaltlich ähnlich gefasstes Reichsinstitut sein sollte, das jedoch nie verwirklicht wurde. ${ }^{69}$ Leiter der Landesstelle war zunächst Karl-Ewald Fritzsch, der als Schüler von Adolf Spamer mit diesem gemeinsam die Landesstelle aufbaute. ${ }^{70}$ An fast allen sächsischen Schulen wurden einzelne Lehrer zu Volkstumswarten ernannt, die einerseits Brauchtumszeugnisse und vorgeblich altgermanisches Kulturgut sammeln sowie auch Brauchtumspflege aktiv betreiben und nicht zuletzt auch neues Brauchtum begründen sollten. Die Erkenntnisse der Landesstelle sollten sich vor allem in Unterricht und Erziehung niederschlagen. Die wabre, lebensvolle Volkstumspflege stand dabei im Vordergrund: Die Landesstelle sei nicht dazu geschaffen worden, um die Zahl der Doktordissertationen zu erböhen, stellte Göpfert fest. ${ }^{71}$ Die sächsische Landesstelle etablierte sich als eines der Zentren der deutschen Volkskunde; die Redaktion der „Mitteldeutschen Blätter für Volkskunde“ wurde nach Dresden verlagert. Auf Initiative Mutschmanns

65 Ansprache Arthur Göpferts vom 10. Februar 1940, in: Rektorwechsel an der Universität Leipzig, Leipzig 1940, S. 37 f.

66 Vgl. PARAK, Hochschule und Wissenschaft (wie Anm. 64), S. 127.

67 Ebd., S. 120, 124.

68 Verordnungsblatt 19 (1937), Nr. 11; Verordnungsblatt 21 (1939), Nr. 8, S. 31.

69 Die deutsche Volkskunde 1 (1934), S. 15; HANS STEGLICH, Praktische Volkstumspflege im Dienste des Heimatwerkes Sachsen, in: Mitteldeutsche Blätter für Volkskunde 13 (1938), H. 1, S. 11. Hans Steglich war Gauwart für Volkstumspflege in der Landesstelle. SCHAARSCHMIDT, Regionalkultur (wie Anm. 20), S. 54. Auch: Wolfgang Jacobeit/ HanNJOST LiXfeld/Olaf BOCKHORN, Völkische Wissenschaft. Gestalten und Tendenzen der deutschen und österreichischen Volkskunde in der ersten Hälfte des 20. Jahrhunderts, Köln/Weimar/Wien 1994.

70 Aufbau der Landesstelle für Volksforschung und Volkstumspflege Sachsen, in: Mitteldeutsche Blätter für Volkskunde 11 (1936), H. 1, S. 23-26. Durch Anordnung der NSDAP-Reichsleitung vom 31. August 1941 bezüglich der Dorfkultur wurde Fritzsch auch zum Gaureferenten für Dorfkultur im NSLB ernannt.

71 HANs STEGLICH, Weg und Ziel praktischer Volkstumsarbeit in der Landesstelle für Volksforschung und Volkstumspflege des NSLB, Gau Sachsen, in: Mitteldeutsche Blätter für Volkskunde 10 (1935), H. 1/2, S. 22-30, hier S. 23. 
entstand 1936 das „Heimatwerk Sachsen - Verein zur Förderung des sächsischen Volkstums e. V.“, das sich u. a. auch der Volkskunde widmete. Das Heimatwerk Sachsen integrierte die Landesstelle für Volksforschung, und Fritzsch wurde innerhalb des Heimatwerkes Referent für Volkskunde.

Wie in der Volkskunde das Verhältnis von Wissenschaft und gelebtem Brauchtum in Teilen neu definiert wurde, unterlagen auch die anderen Wissenschaftsgebiete einer politisch gewollten stärkeren Propaganda, um das ,germanische Erbe' sichtbar werden zu lassen und, wenn möglich, daran aktiv anzuschließen. Für den Dresdner Großen Garten war der Nachbau eines germanischen Gehöfts aus dem 1. Jahrhundert in Originalgröße geplant, wie es in den nordsächsischen Gebieten gestanden haben könnte. ${ }^{72}$ Göpfert unterstützte die Bestrebungen des Museumspflegers Friedrich Lamprecht und des Landespflegers für die Bodenaltertümer, Georg Bierbaum, nach einer zentralen Sammelstelle für Bodenfunde. ${ }^{73}$ Die durch die verstärkten Grabungsaktivitäten im ,Dritten Reich“ zunehmenden Bodenfunde wollten Bürgermeister und Leiter der Heimatmuseen in den jeweiligen Orten belassen. An einer bloßen Stapelung von Scherben in der Provinz kann jedoch einer wirksamen Propaganda nicht gelegen sein, so Lamprecht. ${ }^{74}$ Er widersprach damit dem dezentralen, unwissenschaftlichen Ansatz der Landesstelle Sachsen des ,Propamin' (Propagandaministeriums), die den Aspekt der Propagandierung der Fundstücke im Sinne eines germanischen Anspruchs in den Vordergrund stellen wollte. Die Originale sollten nach Auffassung Lamprechts kopiert und die Faksimiles dann den Heimatsammlungen zur Verfügung gestellt werden. Lamprecht und Bierbaum favorisierten eine gute Auswahl an Kopien in den Heimatmuseen und eine Abkehr vom Prinzip des Alles-Zeigen-Wollens, wie es zu häufig in diesen Museen geschah. Die Bodenfunde sollten in einer Nachbildung des Fundortes ausgestellt werden, um den Anschein einer Topfsammlung und von Polterabendrelikten $\mathrm{zu}$ vermeiden. ${ }^{75}$ Auch das Volksbildungsministerium und Studentkowski hatten sich der Meinung von Lamprechts und Bierbaums angeschlossen.

Wie in anderen Bereichen begaben sich die Nationalsozialisten auch in der Volkskunde in einen Widerspruch. In Sachsen propagierte Mutschmann wie andere Gauleiter auch den Vorrang einer reichsdeutschen Hochsprache, die die mundartlich beeinflusste Umgangssprache ablösen sollte. Schon 1933 hatten Göpfert und der sächsische NSLB der Sprecherziehung einen höheren Stellenwert in der Schule eingeräumt. 1936 begann die anscheinend nur mit Göpfert abgestimmte ,Sachsen-Aktion“Mutschmanns, die einerseits die Verunglimpfung des sächsischen Dialekts durch Literatur und Kabaretts beseitigen, andererseits den Dialekt selbst in seinen ,schlimmsten' Ausprägungen zurückdrängen wollte. ${ }^{76} \mathrm{Zu}$ viele Nachteile schienen die sächsischen Absolventen, im Gegensatz zum Hochdeutschen oder Märkischen, zu haben, war doch der sächsische Dialekt häufig Gegenstand von Satire. Durch die Aussprache und den Sprachklang war er ganz und gar nicht schlicht, knapp und klar, wie die Verordnung forderte - eben preußisch-militärisch sollte die Hochsprache sein, die Mutsch-

72 HStA Dresden, 11125 Ministerium des Kultus und öffentlichen Unterrichts, Nr. 14614 Vorgeschichte in den Heimatmuseen, fol. 11 (Schreiben Lamprecht an Studentkowski vom 6. Juli 1937).

73 Vgl. dazu HStA Dresden, 11125 Ministerium des Kultus und öffentlichen Unterrichts, Nr. 14609 Museumspflege 1935-1943.

74 HStA Dresden, 11125 Ministerium des Kultus und öffentlichen Unterrichts, Nr. 14614 Vorgeschichte in den Heimatmuseen, fol. 3 (Schreiben Lamprecht an Studentkowski vom 6. Juli 1937).

75 Ebd., fol. 12 (Bericht Lamprechts vom 6. Juli 1937).

76 SCHAARSCHMIDT, Regionalkultur (wie Anm. 20), S. 145-148. 
mann und Göpfert an den sächsischen Schulen durchsetzen wollte. ${ }^{77}$ Am 21. Januar 1937 erließ Göpfert eine Verordnung zur „Pflege der deutschen Hochsprache“, die den Sprech- und Rechtschreibunterricht regelte. ${ }^{78}$ Die Hochsprache ist Ausdruck der geistigen Einheit aller Deutschen heißt es dort; die Verordnung fügt sich damit in die ,Überwindung' des sächsischen Dialekts ein. ${ }^{79}$ Auch durch den erwarteten Sieg im Zweiten Weltkrieg kam einer einheitlichen deutschen Hochsprache eine ganz andere Bedeutung zu: Deutsch als Weltsprache musste auch der Rolle Deutschlands als Weltmacht entsprechen, so Göpfert. ${ }^{80}$ Noch im Winter 1942/43 fand die Vorlesungsreihe "Gesetz und Sendung deutscher Sprache“ in Dresden, Chemnitz und Leipzig statt, die die deutsche Spracharbeit im Zeichen des deutschen Sieges thematisierte. ${ }^{81}$

\section{Fazit}

Trotz der Zentralisierungsbestrebungen im Reich gelang Göpfert als Leiter des sächsischen Volksbildungsministeriums eine vergleichsweise selbstständige sächsische Bildungs- und Hochschulpolitik, die sich in Mutschmanns Politik der Machtkonzentration und Kompetenzbewahrung auch im Hinblick auf das Reich einfügte. Seinen Machtbereich baute er auch auf Kosten der anderen Ministerien aus, sodass zu Beginn des Zweiten Weltkrieges die Verantwortung für fast alle sächsischen Bildungs- und Hochschulschuleinrichtungen in seinem Verantwortungsbereich lag. Der Preis dafür war eine erhebliche Indoktrination der Schulen mit nationalsozialistischem Gedankengut, die der NSLB-Funktionär Göpfert vor allem nach seinem Amtsantritt als Leiter des Volksbildungsministeriums 1935 durchsetzte. Zur Unterstützung des zweiten Vierjahresplans rief Göpfert 1937 den Leistungswettkampf sächsischer Schulen aus, der Sachsen zum Land der Musterschulen machen sollte, und zwar sowohl in Leistung und Haltung der Lehrer und der Schüler als auch bei Gebäuden und Innenausstattung. ${ }^{82}$ Die sächsische Pädagogik sollte deutschlandweit vorbildlich sein. Göpfert stellte diesen Wettkampf in den Dienst des Heimatwerks Sachsen. Auch die Hochschulen unterlagen der Politisierung, auch wenn der eigene Anspruch der ,Freiheit der Wissenschaft ${ }^{`}$ dem entgegenstand. Noch stärker als bei den Schulen gelang es bei den Universitäten, die Eigenständigkeit gegenüber dem Reichserziehungsministerium zu bewahren.

77 Wege zur Musterschule, hrsg. vom Ministerium für Volksbildung, Dresden 1937 (= Beilage zu: Politische Erziehung).

78 Verordnungsblatt 19 (1937), Nr. 3, S. 12. 1939 Richtlinien für den Deutschunterricht der Volksschule

79 Verordnungsblatt 19 (1937), Nr. 3, S. 12. Vgl. auch: GöTz, Grundschule (wie Anm. 50), S. 92-95.

80 So Göpfert an die Oberstudiendirektoren, Direktoren, Bezirksschulämter und Lehrgangsleiter am 1. April 1942, nach: SCHAARSCHMIDT, Regionalkultur (wie Anm. 20), S. 218.

81 Verordnungsblatt 24 (1942), Nr. 28, S. 138.

82 Verordnungsblatt 19 (1937), Nr. 3, S. 11. 